\title{
EIGENMODES OF ISOSPECTRAL DRUMS
}

\author{
TOBIN A. DRISCOLL *
}

\begin{abstract}
Recently it was proved that there exist nonisometric planar regions that have identical Laplace spectra. That is, one cannot "hear the shape of a drum." All known examples of such regions are bounded by polygons with reentrant corners. While the isospectrality can be proven mathematically, analytical techniques are unable to produce the eigenvalues themselves. Furthermore, standard numerical methods for computing the eigenvalues, such as adaptive finite elements, are highly inefficient. Physical experiments have been performed to measure the spectra, but the accuracy and flexibility of this method are limited. We describe an algorithm due to Descloux and Tolley that blends finite elements with domain decomposition, and show that, with a modification that doubles its accuracy, this algorithm can be used to compute efficiently the eigenvalues for polygonal regions. We present results accurate to twelve digits for the most famous pair of isospectral drums, as well as results for another pair.
\end{abstract}

Key words. eigenvalues, elliptic operators, isospectrality, finite-element methods, domain decomposition, method of particular solutions

AMS subject classifications. $65 \mathrm{~N} 25,35 \mathrm{P} 99,35 \mathrm{Q} 60$

1. Introduction. In 1966 Mark Kac [13] posed the question, "Can one hear the shape of a drum?" He was referring to the problem of whether the Laplacian operator with Dirichlet boundary conditions could have identical spectra on two distinct planar regions. Recently Gordon, Webb, and Wolpert [12] answered the question negatively by an elegantly constructed counterexample, justifiably attracting a great deal of attention $[7,8,16]$. The simplest form of their example is a pair of regions bounded by eight-sided polygons, henceforth called the GWW isospectral drums; see Figure 1. Numerous similar examples have since been discovered [5], all in the form of nonconvex polygons [4].

The simplest and most versatile proof of isospectrality employs "transplantation" [3] of the eigenfunctions. The polygons are shown to be (or are constructed to be) made up of nonoverlapping translations, rotations, and reflections of a single shape, such as a triangle. Given an eigenfunction on one region, one can prescribe a function over the other region whose values over each piece are linear combinations of the eigenfunction values over several of the pieces of the first region. The combinations are chosen to satisfy the boundary conditions and to match values and derivatives at interfaces between pieces, and the interior equation is satisfied by superposition. Hence the result is an eigenfunction of the second region having the same eigenvalue. To complete the proof of isospectrality, one need only check that the procedure is invertible. Note that the proof is nonconstructive and other information, particularly the actual values of the eigenvalues, remains unknown.

To find the eigenvalues, it is natural to turn to numerical computation. However, standard numerical procedures for computing the eigenvalues [15] appear to be surprisingly inefficient for this problem. For example, we shall show that PLTMG [2], an adaptive finite element package written by Bank, obtains very slow convergence for the eigenvalue estimates. Another well-known technique for eigenvalue problems, the method of particular solutions [11], fails to produce estimates of accuracy better than a few percent.

* Center for Applied Mathematics, Cornell University, Ithaca, NY 14853. E-mail to driscoll@na-net.ornl.gov. 

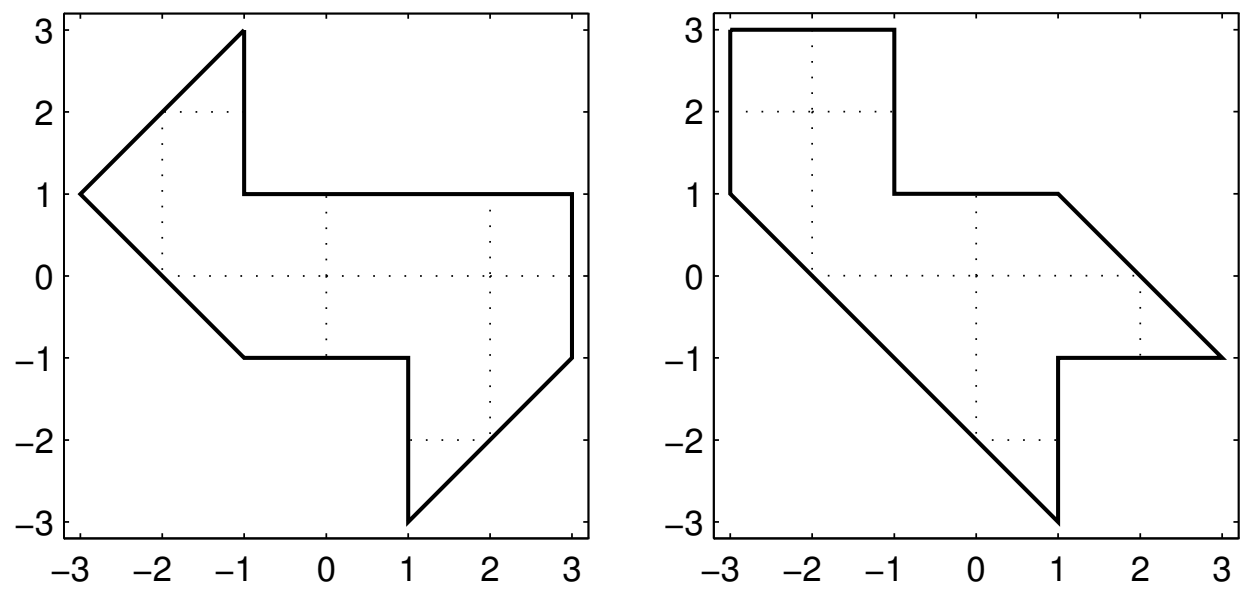

FIG. 1. The GWW isospectral drums, and subdivisions used for the domain decomposition method.

The first successful determination of the spectra of the GWW drums was by Sridhar and Kudrolli [17], who used a physical approach. They constructed microwave cavities in the shapes of the polygons and measured resonances in transverse magnetic waves, which obey the Helmholtz equation. In this manner they have obtained the first 54 eigenvalues to an accuracy of about $0.3 \%$. Of course, this method is not practical for experiments with many different regions.

More recently, Wu, Sprung, and Martorell [18] used a mode matching numerical approach to compute 25 eigenvalues of the GWW drums, and compared their results to values extrapolated from finite difference calculations. Their mode matching approach is efficient, but it depends on the region being decomposed into simple shapes for which all the eigenmodes can be explicitly written in closed form. We shall show that the numbers computed by $\mathrm{Wu}$ et al. are accurate to about four digits.

A little-known numerical method due to Descloux and Tolley [9] is intended specifically for eigenvalue computations on polygons. This algorithm, which is a combination of domain decomposition and finite element methods, is applicable to any planar polygon and can efficiently compute eigenvalues to an accuracy on the order of the square root of machine precision. With a small modification, the accuracy of the method can be improved to the order of machine precision. Using this improved method, we have computed the first 25 eigenvalues of the GWW isospectral drums to an accuracy of at least 12 digits. Each eigenvalue calculation takes a few minutes on a workstation, and eigenfunctions can be computed just as quickly at arbitrary domain points.

In this paper we describe some of the limitations of the standard numerical approaches to computing the eigenvalues of the GWW drums. We then outline the domain decomposition method of Descloux and Tolley and describe our accuracydoubling modification. We present numerical and graphical results of eigenvalue calculations for the GWW drums, comparing our results to the previously published estimates described above. A comparison is made of the method's efficiency with that of the finite element package PLTMG. We also present the results of this method applied to another pair of polygonal isospectral drums. 
2. Algorithms. Given a planar region $\Omega$ with polygonal boundary $\partial \Omega$, our goal is to find approximations to one or more eigenpairs $(\lambda, u) \in\left(\mathbf{R}^{+}, C(\bar{\Omega})\right)$ satisfying

$$
\begin{aligned}
\Delta u+\lambda u=0 & \text { in } \Omega, \\
u=0 & \text { on } \partial \Omega .
\end{aligned}
$$

A direct numerical approach to this problem is to use a finite element software package. We chose PLTMG [2] because of its widespread availability and automatic adaptive mesh refinement capabilities. PLTMG regards the linear problem (1) as a nonlinear continuation problem with parameter $\lambda$ and functional $\rho(u)=\|u\|_{L^{2}}$. The procedure, which is outlined in Section 4.6.2 of [2], is to track the zero solution for varying $\lambda$ until a bifurcation point in the $\lambda$ - $\rho$ plane is found, at which point the bifurcating branch with constant $\lambda$ (the eigenfunction) is followed. The grid is adaptively improved and the estimate for $\lambda$ updated until the desired accuracy is apparently achieved.

The principal disadvantage of using PLTMG for this problem is the loss of regularity in the solutions near corners in $\Omega$, particularly reentrant corners. Even though PLTMG selectively refines the mesh near the reentrant corners as the iteration proceeds, convergence to the correct eigenvalues is slow.

While the singularity of an eigenfunction at a corner can be severe, its precise form is very well known. If $(u, \lambda)$ is an eigenpair, and $(r, \phi)$ are suitably oriented polar coordinates originating from a corner of $\partial \Omega$ with interior angle $\pi / \alpha$, then

$$
u(r, \phi)=\sum_{n=1}^{\infty} c_{n} J_{n \alpha}(\sqrt{\lambda} r) \sin (n \alpha \phi)
$$

where $J_{\nu}$ is a Bessel function of the first kind. This expression, which is essentially just a Fourier series, is valid at least for $r$ less than the distance to the nearest other corner of $\partial \Omega$. To be efficient, an algorithm for the problem (1) ought to make use of this expansion.

One approach to exploiting this information is the method of particular solutions, or point matching [15], introduced by Fox, Henrici, and Moler [11], who illustrated its use with an L-shaped region. ${ }^{1}$ This method truncates the expansion (2) taken about the reentrant corner(s) and determines eigenvalues by requiring the trial solution to be zero at collocation points along the boundary $\partial \Omega$. In practice, this reduces to detecting singularity in a matrix which depends nonlinearly on a parameter $\lambda$. However, as the authors note, this method does not work well for regions with more than one reentrant corner, and our experience bears this out. The difficulty is that as the number of terms in the truncated expansion is increased, the matrix becomes very nearly singular for all values of $\lambda$, and detecting the true singularity numerically becomes impossible. In fact, we have been unable to produce more than 2 or 3 accurate digits for a few of the smallest eigenvalues with this method, even after including expansions about all of the corners.

The underlying source of difficulty in the method of particular solutions is the global treatment of the local eigenfunction expansion (2). To take a more local approach, let the polygon $\Omega$ be subdivided into several nonoverlapping pieces $\Omega_{j}$, $j=1, \ldots, N$. We denote each interface $\partial \Omega_{j} \cap \partial \Omega_{k}$, which may be empty, by $\Gamma_{j k}$.

1 Their calculations are the basis of the logo of The MathWorks, Inc., and can be demonstrated with the membrane command in MATLAB. 
Suppose that, given a scalar $\lambda$, we can find a set of $N$ "subfunctions" $u_{j} \in C\left(\overline{\Omega_{j}}\right)$ such that $\left(\lambda, u_{j}\right)$ is a certain eigenpair on subregion $\Omega_{j}$ :

$$
\begin{aligned}
\Delta u_{j}+\lambda u_{j}=0 & \text { in } \Omega_{j}, \\
u_{j}=0 & \text { on } \partial \Omega \cap \partial \Omega_{j} .
\end{aligned}
$$

(Note the difference between boundary conditions (1b) and (3b).) It is well known that $\lambda$ is an eigenvalue for the whole region $\Omega$ if and only if along each nonempty interface $\Gamma_{j k}$, the subfunctions $u_{j}$ and $u_{k}$ and their normal derivatives match continuously. This fact is at the root of the transplantation proof of isospectrality, and an analogous observation underlies many domain decomposition methods from the numerical solution of elliptic PDEs [6].

The method of mode matching described by Wu, Sprung, and Martorell [18] is one way to exploit this idea. In this method, the expansion (2) is not used. Instead, analytic expressions of the solutions to (3) must be known throughout each subdomain $\Omega_{j}$. For the GWW drums of Figure 1, it is possible to accomplish this by dividing each drum into five pieces, each of which is a square or a $\left(45^{\circ}, 45^{\circ}, 90^{\circ}\right)$ triangle. Because of the simple shapes, the eigenfunctions are differences between products of sine functions. A subfunction $u_{j}$ is expanded as a combination of these functions with unknown coefficients, the expansions are truncated, and the requirement of functions and derivatives matching at interfaces becomes a linear system in these coefficients. As with the method of particular solutions, an eigenvalue is a value of $\lambda$ for which the matrix of this system becomes singular. In this case, however, Wu et al. report no difficulty with numerical near-singularity.

A shortcoming of the mode matching method is that it is not universally applicable. In general we cannot expect $\Omega$ to admit a simple decomposition for which the eigenfunctions of the individual pieces can be explicitly written in a convenient and usable form. A universal algorithm ought to return to the expansion (2), which is always available.

To this end, let us assume that the boundary of each $\Omega_{j}$ includes a portion of the boundary of the whole region $\Omega$ in such a way that exactly one vertex $V_{j}$ with interior angle $\theta_{j}$ of the original polygon is in $\partial \Omega_{j}$; if necessary, extra vertices are added to $\partial \Omega$ with $\theta_{j}=\pi$. In Figure 1 we illustrate one such subdivision for the GWW isospectral polygons.

We could now enforce the matching conditions at collocation points on the interfaces, in the manner of the method of particular solutions. However, Descloux and Tolley [9] find the familiar problem with location of the singularity of the resulting matrix, and instead propose a method that employs finite element ideas within the domain decomposition framework. At the heart of their algorithm are the functionals

$$
\begin{aligned}
& R\left(\lambda ; u_{1}, \ldots, u_{N}\right)=\sum_{j<k} \int_{\Gamma_{j k}}\left[\left(u_{j}-u_{k}\right)^{2}+\left|\nabla u_{j}-\nabla u_{k}\right|^{2}\right] d s \\
& M\left(\lambda ; u_{1}, \ldots, u_{N}\right)=\sum_{j=1}^{N} \iint_{\Omega_{j}} u_{j}^{2} d x d y .
\end{aligned}
$$

For fixed $\lambda$, let $\mu(\lambda)$ be the minimum of the quotient $R / M$ over all choices of subfunctions. Now $\mu(\lambda)=0$ if and only if $\lambda$ is an eigenvalue of (1) and each $u_{j}$ is the restriction of the eigenfunction $u$ to $\Omega_{j}{ }^{2}$

${ }^{2}$ Descloux and Tolley were able to prove convergence of their algorithm only when gradients, 

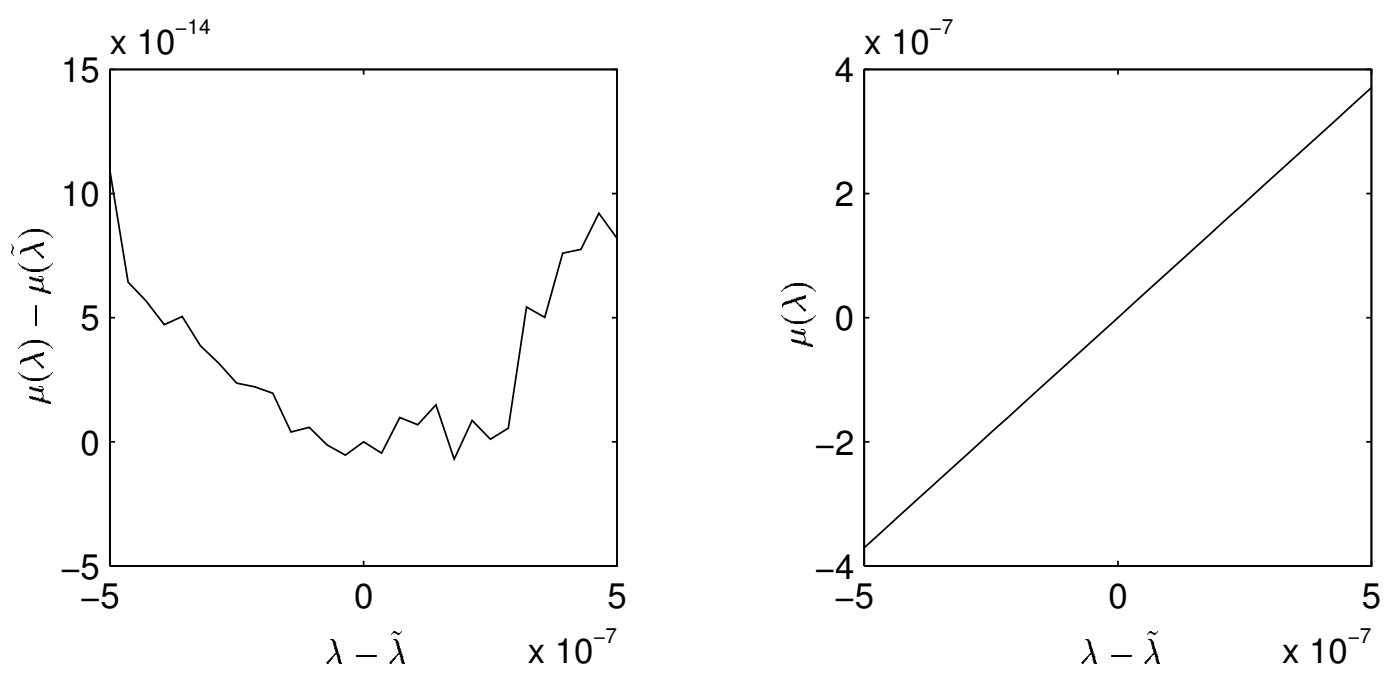

FIG. 2. Comparison of $\mu(\lambda)$ with $\dot{\mu}(\lambda)$ near a minimum $\tilde{\lambda}$, which is an estimate of the first $G W W$ eigenvalue.

We now use the finite element idea of replacing a minimization over infinitedimensional function spaces by minimization over a nested family of finite-dimensional approximations. As bases for these spaces we choose terms of the local Fourier-Bessel expansions; that is, each subfunction $u_{j}$ is expressed as a combination of $n_{j}$ terms of the expansion (2) about $V_{j}$. This guarantees that the subproblem (3) is satisfied, even when $\lambda$ is not an eigenvalue of $\Omega .^{3}$ For optimal performance, $n_{j}$ should be proportional to $\theta_{j}$, the interior angle at $V_{j}$.

The corresponding approximation to $\mu(\lambda)$ now becomes the solution to a generalized matrix eigenproblem:

$$
A(\lambda) v(\lambda)=\mu(\lambda) B(\lambda) v(\lambda) .
$$

The matrix $A$ is computed by evaluating (4) by Gauss-Legendre quadrature with $q$ nodes on each interface, and (5) is approximated by integrals over circular sectors so as to make the mass matrix $B$ diagonal. This diagonality makes it convenient to replace the generalized eigensystem by the standard eigenproblem for $B^{-1 / 2} A B^{-1 / 2}$. Finally, a value of $\lambda$ which minimizes our approximation to $\mu$ is taken as an estimate of an eigenvalue of (1a)-(1b).

Here is where we improve upon Descloux and Tolley's original algorithm. Suppose we can compute $\mu$ only to accuracy $\epsilon$, which is on the order of machine precision. Because of the quadratic nature of $\mu$ near a minimum, a straightforward minimization gives an accuracy in $\lambda$ of only order $\sqrt{\epsilon}$. If instead we seek solutions to $\dot{\mu}(\lambda)=0$, the linearity of $\dot{\mu}$ near a minimum allows us to find $\lambda$ to an accuracy comparable to that of $\mu$. Figure 2 illustrates the situation for an estimate $\tilde{\lambda}$ of the first eigenvalue of the

rather than normal derivatives, appear in (4).

3 Actually, $\partial \Omega \cap \partial \Omega_{j}$ may contain isolated points where the boundary condition (3b) is not explicitly satisfied. However, the matching conditions do enforce this condition, and experiments show that slight changes in the $\Omega_{j}$ that avoid this problem do not substantially affect the performance of the algorithm. 


\begin{tabular}{|lllll|}
\hline 2.53794399980 & 9.20929499840 & 14.3138624643 & 20.8823950433 & 24.6740110027 \\
3.65550971352 & 10.5969856913 & 15.8713026200 & 21.2480051774 & 26.0802400997 \\
5.17555935622 & 11.5413953956 & 16.9417516880 & 22.2328517930 & 27.3040189211 \\
6.53755744376 & 12.3370055014 & 17.6651184368 & 23.7112974848 & 28.1751285815 \\
7.24807786256 & 13.0536540557 & 18.9810673877 & 24.4792340693 & 29.5697729132 \\
\hline
\end{tabular}

TABLE 1

The first twenty-five eigenvalues of the $G W W$ isospectral drums. We believe that the numbers are correct to all digits shown.

GWW drums. By differentiating (6) with respect to $\lambda$ and left-multiplying through by $v^{T}$, we see that

$$
\dot{\mu}(\lambda)=\frac{v^{T}(\dot{A}-\mu \dot{B}) v}{v^{T} B v} .
$$

The matrices $\dot{A}$ and $\dot{B}$ can be computed in a straightforward manner.

To summarize, the algorithm can be viewed as an iteration in the parameter $\lambda$ whose convergence is dictated by domain decomposition considerations. Each step of the iteration is computed approximately by a large singular finite element method, where the basis functions depend nonlinearly on the parameter $\lambda$. Improved accuracy is achieved by increasing the number of basis functions in the inner step, as with $p$-type finite element methods.

3. Results. In Table 1 we list our estimates of the first 25 eigenvalues of the GWW isospectral drums. For these calculations we used $n_{j}=36 / \alpha_{j}=36 \theta_{j} / \pi$ basis functions in region $\Omega_{j}$ and $q=40$ Gauss quadrature points on each interfacial line segment. The results for the two drums agree with each other nearly to machine precision.

In Figure 3 we compare $N(\lambda)$, the number of eigenvalues (counting multiplicity) less than $\lambda$, to the corrected Weyl's formula, which for a polygon is [1]

$$
N(\lambda) \approx \frac{A}{4 \pi} \lambda-\frac{P}{4 \pi} \sqrt{\lambda}+\sum_{j=1}^{N} \frac{1}{24}\left(\alpha_{j}-\alpha_{j}^{-1}\right),
$$

where $A$ is the area of the region $\Omega$ and $P$ is its perimeter. (By isospectrality, $A$ and $P$ are necessarily the same for the two drums.) The formula agrees excellently with the exact $N(\lambda)$.

We believe that the entries of Table 1 are accurate to all digits shown. As support for this claim, in Figure 4 we present the convergence history of the estimates with respect to $n_{j}=4 m / \alpha_{j}$. For each value of $m$, we find that the estimates of any eigenvalue for the two drums agree essentially to machine precision, and we use $\lambda^{(m)}$ to denote this common number. Figure 4 shows the relative change in the successive estimates $\lambda^{(m)}$ as $m$ varies. The rate of convergence is governed by the parameter identified in [9]:

$$
\omega=\max _{j}\left[\left(\frac{\max _{z \in \Omega_{j}}\left|z-V_{j}\right|}{\min _{k \neq j}\left|V_{k}-V_{j}\right|}\right)^{\left(n_{j} / m\right) \alpha_{j}}\right] .
$$

Here, $\omega=1 / 4$. However, the observed convergence is like $\omega^{2 m}$, better than the upper bound of $\omega^{m}$ that Descloux and Tolley were able to prove. In general, curves which 


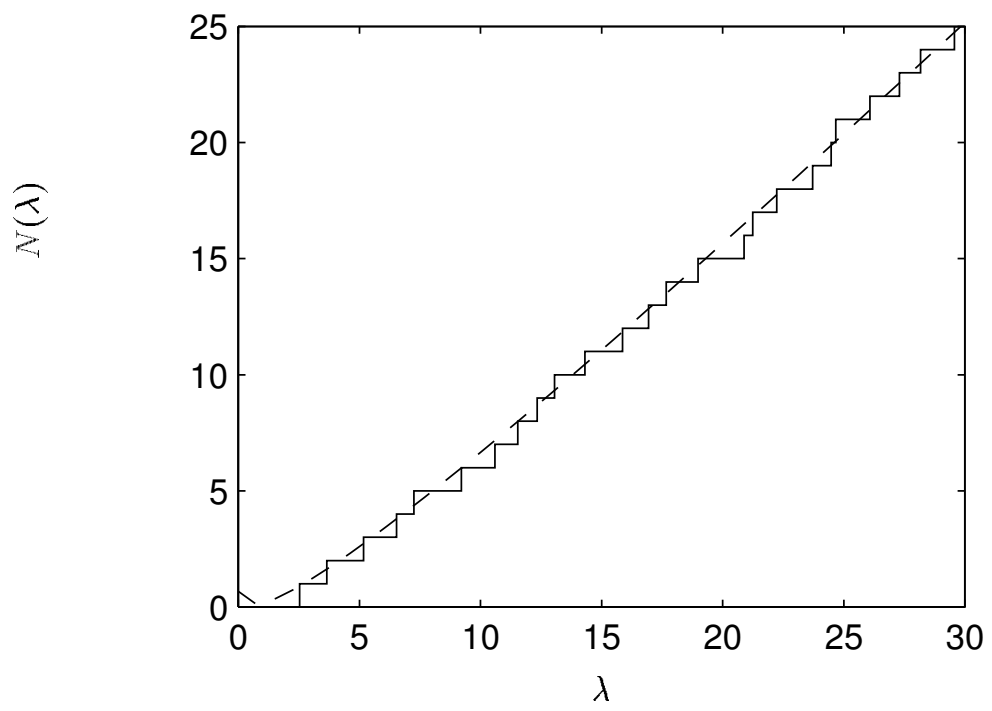

FIG. 3. Integrated eigenvalue density for the $G W W$ drums. The solid stairstep is the actual density, compared with the dashed line representing Weyl's formula (8).

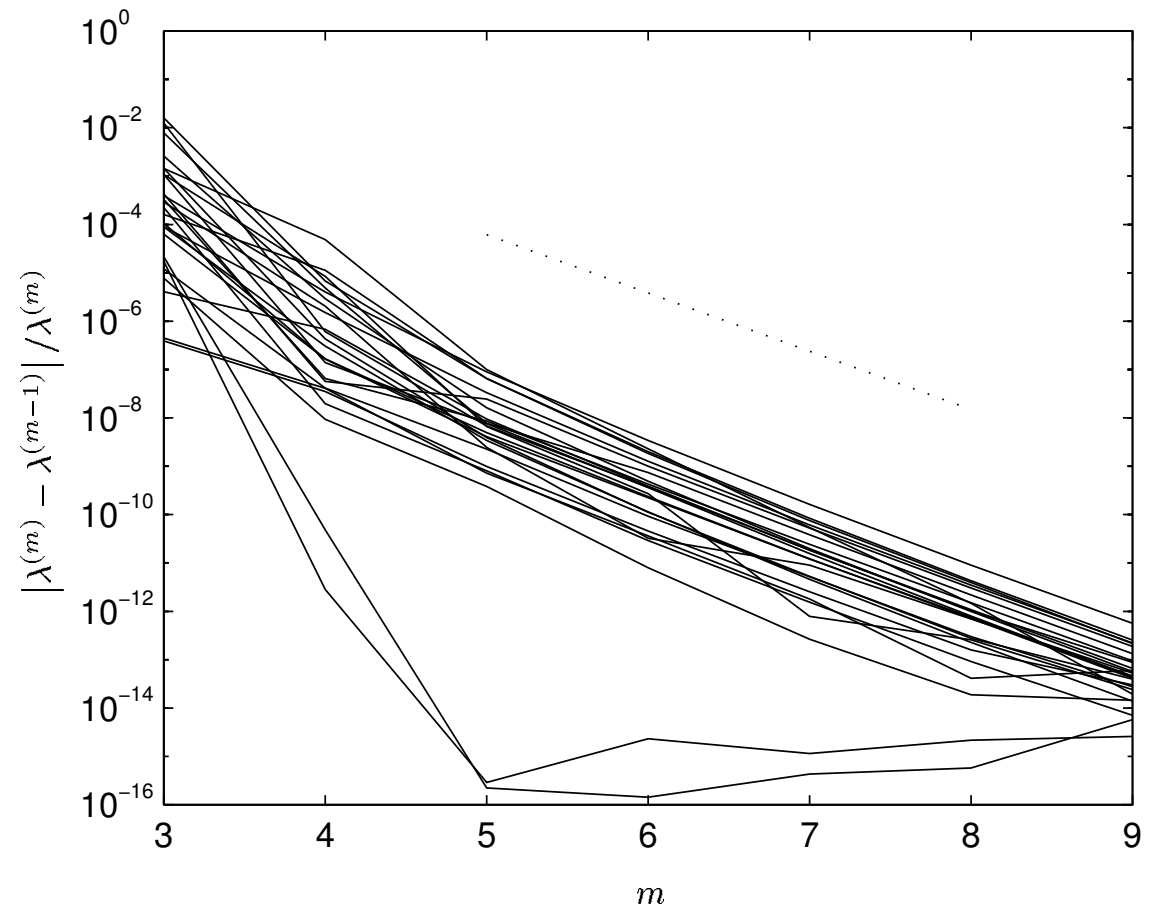

FIG. 4. Convergence of the eigenvalue estimates. The dotted line shows $\omega^{2 m}$, with $\omega$ given by (9). See text for comments about the two dramatically decreasing curves. 
are higher on the graph correspond to higher eigenvalues, the two "superconvergent" curves being exceptions which are discussed below. Note that all the convergence curves end at less than $10^{-12}$.

As mentioned previously, a feature of the results is the dramatic agreement for all the estimates of the two drums, regardless of their accuracy. In fact, all the eigenvalues of the generalized system (6), and hence $\mu(\lambda)$, are numerically identical for the two regions for any $m$. Presumably this occurs because the subdivisions of Figure 1 respect the transplantation symmetries between the regions. As a further check on our results, we applied the algorithm using the less regular subdivisions, selected arbitrarily, depicted in Figure 5. The estimates for the two regions now differ by amounts consistent with their apparent accuracy. In Figure 6 we compare the difference between corresponding values at each $m$ to the rate $\omega^{2 m}$, where now $\omega \approx 0.52$. The estimates for $m=11$ all agree with the numbers of Table 1 to the full 12 digits.

Figures $7-8$ show in detail the first eight eigenfunctions of the GWW drums, including the nodal lines. For comparison, in Figure 9 we reproduce the results of microwave measurements made by Sridhar and Kudrolli [17] for modes 1, 3, and 6 . The microwave results, while noisy, do recognizably represent the shapes of the modes.

Figure 10 shows contours for the ninth mode, which is clearly equivalent to the first mode on a $\left(45^{\circ}, 45^{\circ}, 90^{\circ}\right)$ triangle. This triangle is the fundamental shape that forms the basis of the transplantation proof. The exact eigenvalue in this case is $5 \pi^{2} / 4$, which agrees with our computed values to 15 digits. A similar phenomenon occurs at the twenty-first mode, which is equivalent to the second mode on the triangle with eigenvalue $10 \pi^{2} / 4$. In fact, these two modes account for the "superconvergent" curves of Figures 4 and 6 . We hypothesize that the accelerated convergence occurs because the symmetries of these eigenfunctions about the corners cause many of the Fourier coefficients in (2) to be exactly zero.

In Figure 11 we compare our results to other published determinations of the first 25 eigenvalues. Based on their microwave experiments, Sridhar and Kudrolli report these eigenvalues to an rms relative accuracy of about $0.3 \%$. We observe that the error in their estimates is frequently much larger than the agreement between their values, but there is no clear explanation for this phenomenon [14]. The eigenvalues obtained by Wu, Sprung, and Martorell [18] by extrapolation of results from finite differences and mode matching agree with our results to about 3 and 4 digits, respectively. ${ }^{4}$

In Figure 12 we compare the efficiency of the domain decomposition method to computation of the eigenvalues in PLTMG as described in Section 2. Clearly PLTMG is not competitive for this problem. Figure 13 shows the adaptive grid process for the first eigenvalue on the first drum. The finest structure occurs near the corner where the eigenfunction is large. At this stage, there are 966 triangles and 402 vertices, and the eigenvalue estimate is about 2.5659 .

One advantage of the domain decomposition method over physical experiment and mode matching is its flexibility in application to other polygons. We have applied the domain decomposition method to another pair of isospectral drums, depicted in Figure 14. These regions were constructed using the techniques of Buser et al. [5]. The fundamental unit of the construction is a $\left(30^{\circ}, 70^{\circ}, 80^{\circ}\right)$ triangle, which renders the mode matching method impractical. The first ten eigenvalues of these regions

\footnotetext{
4 In the mode matching method, the degenerate modes 9 and 21 are not computed but are taken exact.
} 


\begin{tabular}{|ll|}
\hline 5.63126379 & 18.8537757 \\
7.18148848 & 19.8509471 \\
12.7905748 & 24.1803291 \\
13.0935554 & 27.5379471 \\
17.0680091 & 30.0098327 \\
\hline
\end{tabular}

TABLE 2

First ten eigenvalues of the isospectral drums of Figure 14.

are listed in Table 2 to nine digits. In Figure 15 we present the convergence history analogous to Figure 4. Here, the value of $\omega$ is about 0.48 , reflecting slower convergence. Selected eigenfunctions for these drums are displayed in Figure 16.

4. Conclusions. Elliptic problems on regions with corners, especially reentrant corners, are recognized as numerically difficult. Indeed, general-purpose packages such as PLTMG do not use known information about the singularities of solutions near the corners, and the method of particular solutions uses this information only globally, restricting its usefulness. Occasionally one can use methods such as mode matching to exploit special geometries. However, the mixed finite element-domain decomposition method exploits the corner information in an appropriately local and completely general fashion, resulting in an efficient and accurate algorithm for the eigenvalues of arbitrary polygonal regions. Using this algorithm, we have made the first high-precision determinations of the eigenvalues of the GWW drums. We have also demonstrated that the method is flexible enough to be applied to other instances of polygonal isospectral regions.

To see more of the eigenfunctions of the GWW drums, and some animations of vibrations arising from selected combinations of the modes, use a World-Wide Web browser such as Mosaic to open the URL http://cam.cornell.edu/ ${ }^{\mathrm{d}}$ riscoll/.

5. Acknowledgments. I would like to thank David Webb, Peter Doyle, Jean Descloux, S. Sridhar and Arshad Kudrolli for their cooperation and pointers to relevant literature. Figure 9 from the microwave experiments was supplied by Sridhar and Kudrolli. I am also grateful to Steve Vavasis and Nick Trefethen for their valuable comments and suggestions.

This work was supported by DOE grant DE-FG02-94ER25199.

\section{REFERENCES}

[1] H. P. Baltes AND E. R. Hilf, Spectra of Finite Systems, Bibliographisches Institut, Mannheim, 1976.

[2] R. BAnk, PLTMG Users' Guide 7.o: A Software Package for Solving Elliptic Partial Differential Equations, SIAM, 1994.

[3] P. BÉRARD, Transplantation et isospectralité, Math. Ann., 292 (1992), pp. 547-559.

[4] P. Buser. private communication, Jan. 1995.

[5] P. Buser, J. Conway, P. Doyle, and K. Semmler, Some planar isospectral domains, Int. Math. Res. Not., (to appear).

[6] T. F. Chan and T. P. Mathew, Domain decomposition algorithms, Acta Numerica, (1994), pp. 61-143.

[7] S. J. Chapman, Drums that sound the same, Amer. Math. Mon., 102 (1995), pp. 124-138.

[8] B. Cipra, You can't hear the shape of a drum, Science, 255 (1992), pp. 1642-1643.

[9] J. Descloux And M. Tolley, An accurate algorithm for computing the eigenvalues of a polygonal membrane, Comp. Meth. App. Mech. Eng., 39 (1983), pp. 37-53. 
[10] N. H. Fletcher and T. D. Rossing, The Physics of Musical Instruments, Springer-Verlag, 1991.

[11] L. Fox, P. Henrici, And C. Moler, Approximations and bounds for eigenvalues of elliptic operators, SIAM J. Num. Analy., 4 (1967), pp. 89-102.

[12] C. Gordon, D. WebB, And S. Wolpert, Isospectral plane domains and surfaces via Riemannian orbifolds, Invent. Math., 110 (1992), pp. 1-22.

[13] M. KAC, Can one hear the shape of a drum?, Amer. Math. Mon., 73 part II (1966), pp. 1-23.

[14] A. Kudrolli. private communication, Feb. 1995.

[15] J. R. Kuttler And V. G. Sigillito, Eigenvalues of the Laplacian in two dimensions, SIAM Review, 26 (1984), pp. 163-193.

[16] I. Peterson, Beating a fractal drum: How a drum's shape affects its sound, Science News, 146 (1994), pp. 184-185.

[17] S. SRidhar and A. Kudrolli, Experiments on not "hearing the shape" of drums, Phys. Rev. Let., 72 (1994), pp. 2175-2178.

[18] H. Wu, D. W. L. Sprung, And J. Martorell, Numerical investigation of isospectral cavities built from triangles, Phys. Rev. E, 51 (1995), pp. 703-708. 

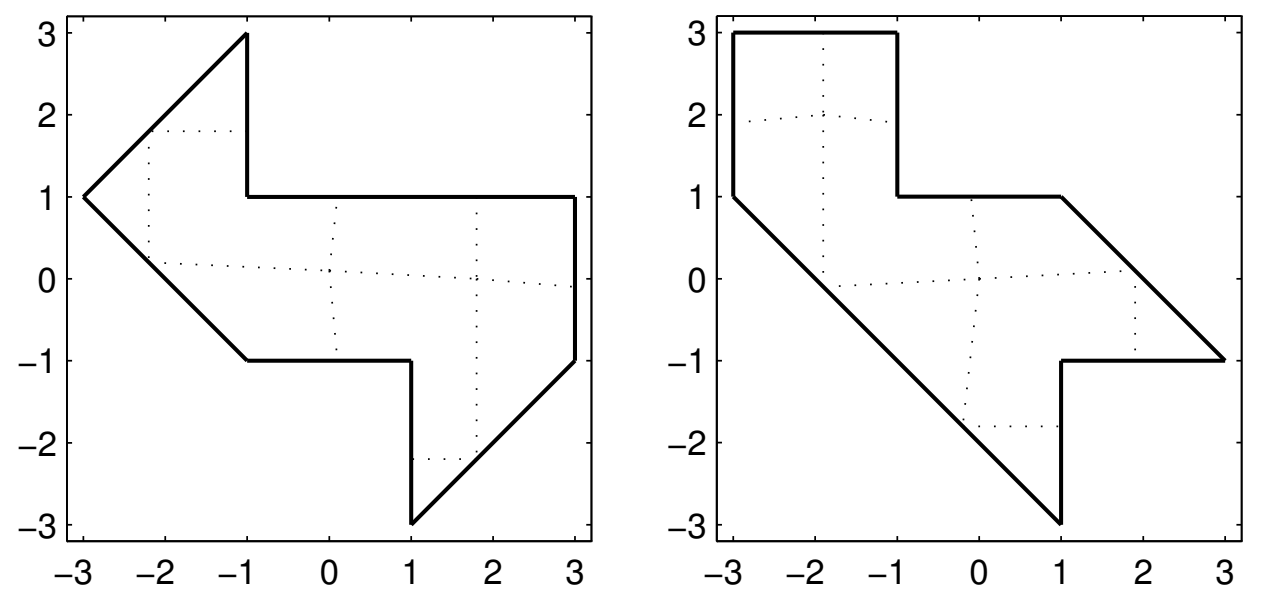

FIG. 5. An alternate subdivision of the $G W W$ drums.

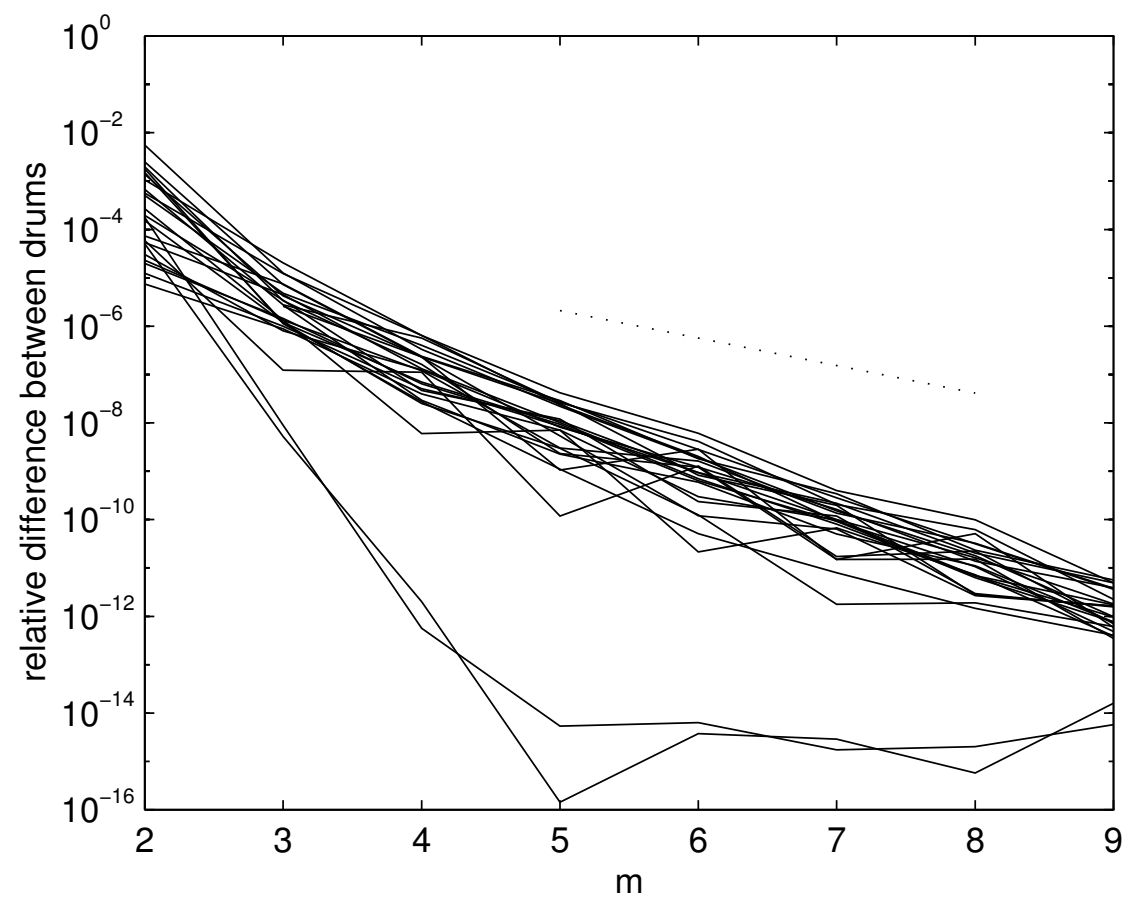

FIG. 6. Difference in estimates for the two drums at each $m$ when using the subdivisions of Figure 5. 

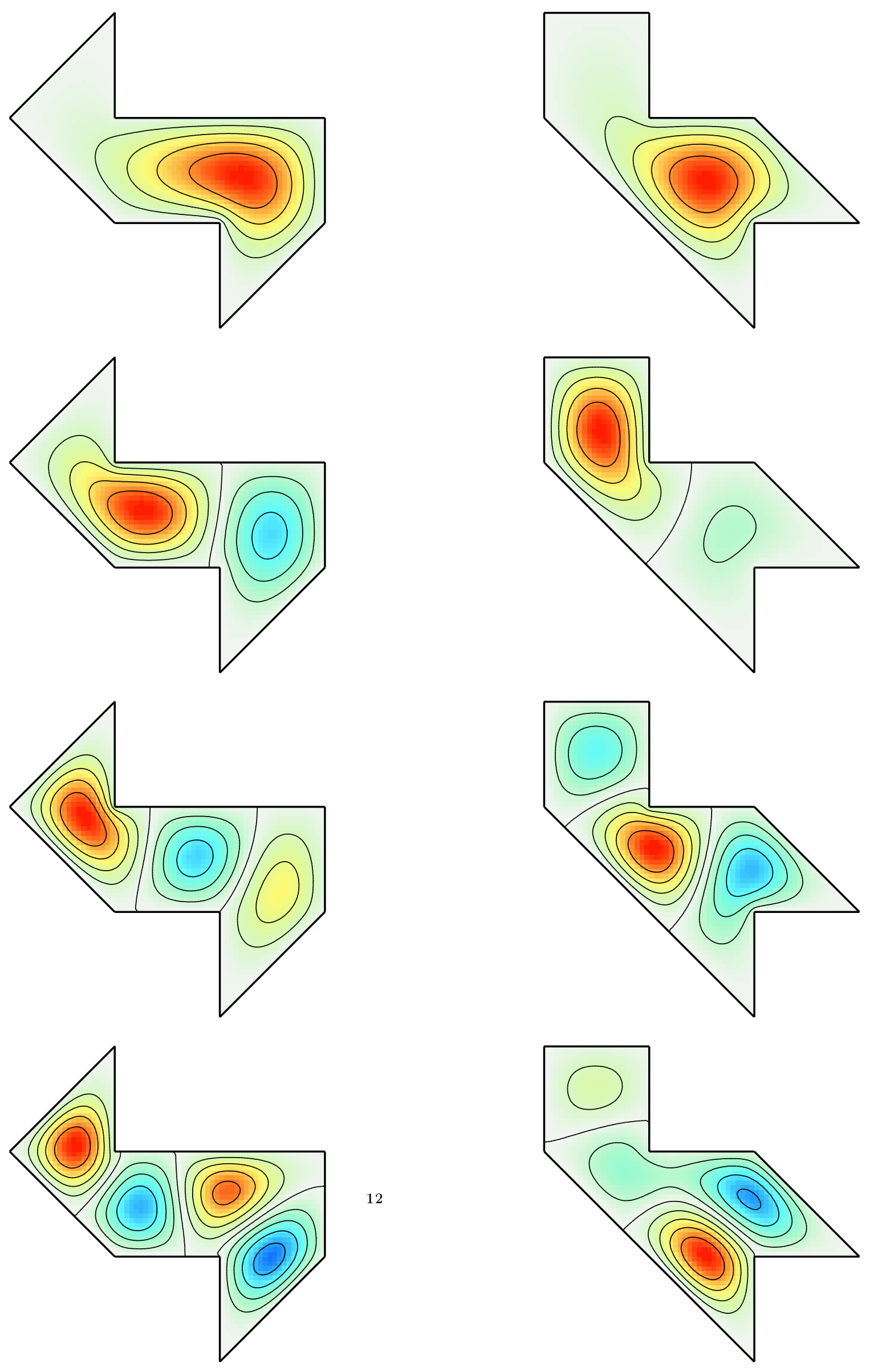

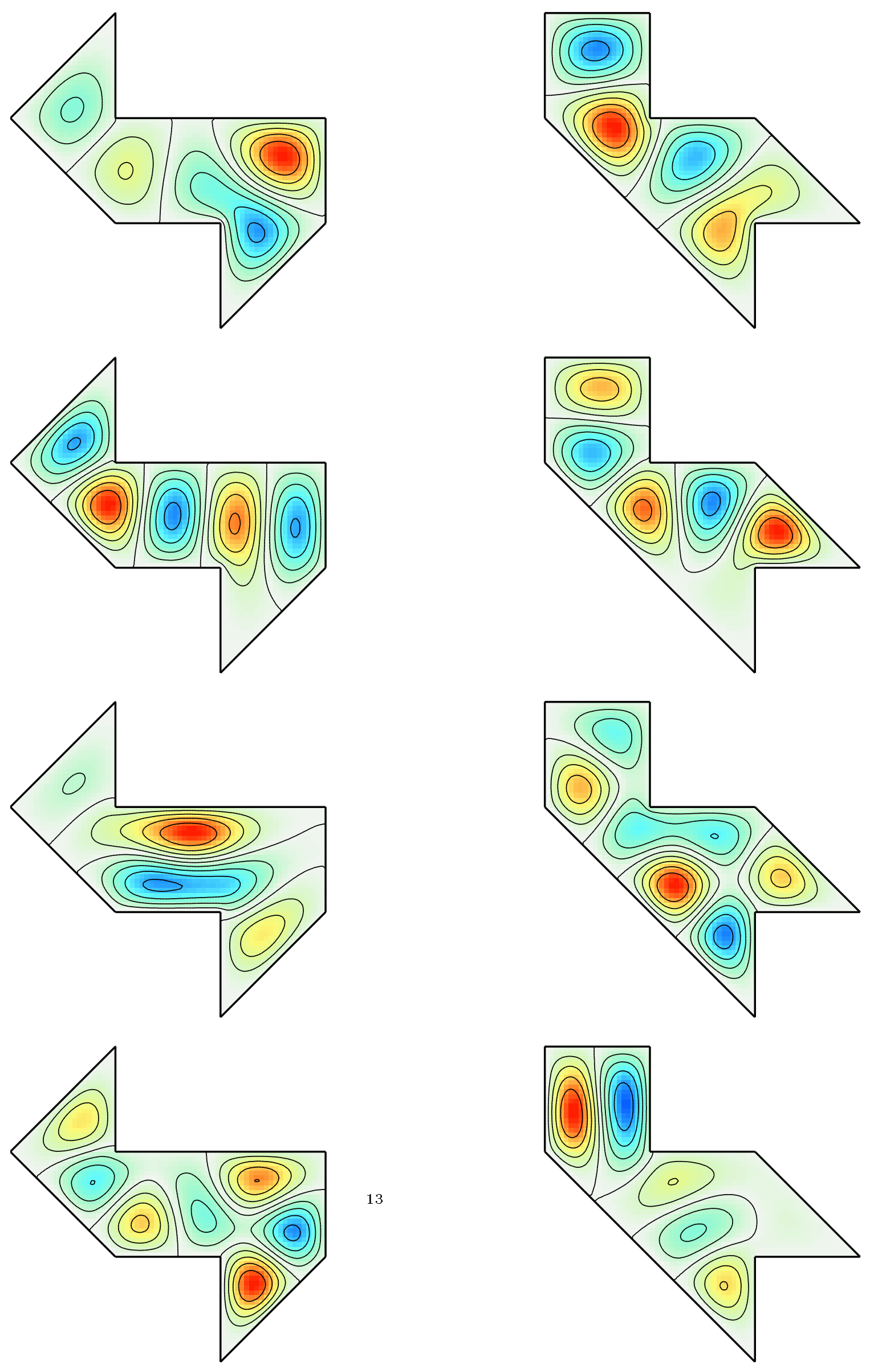

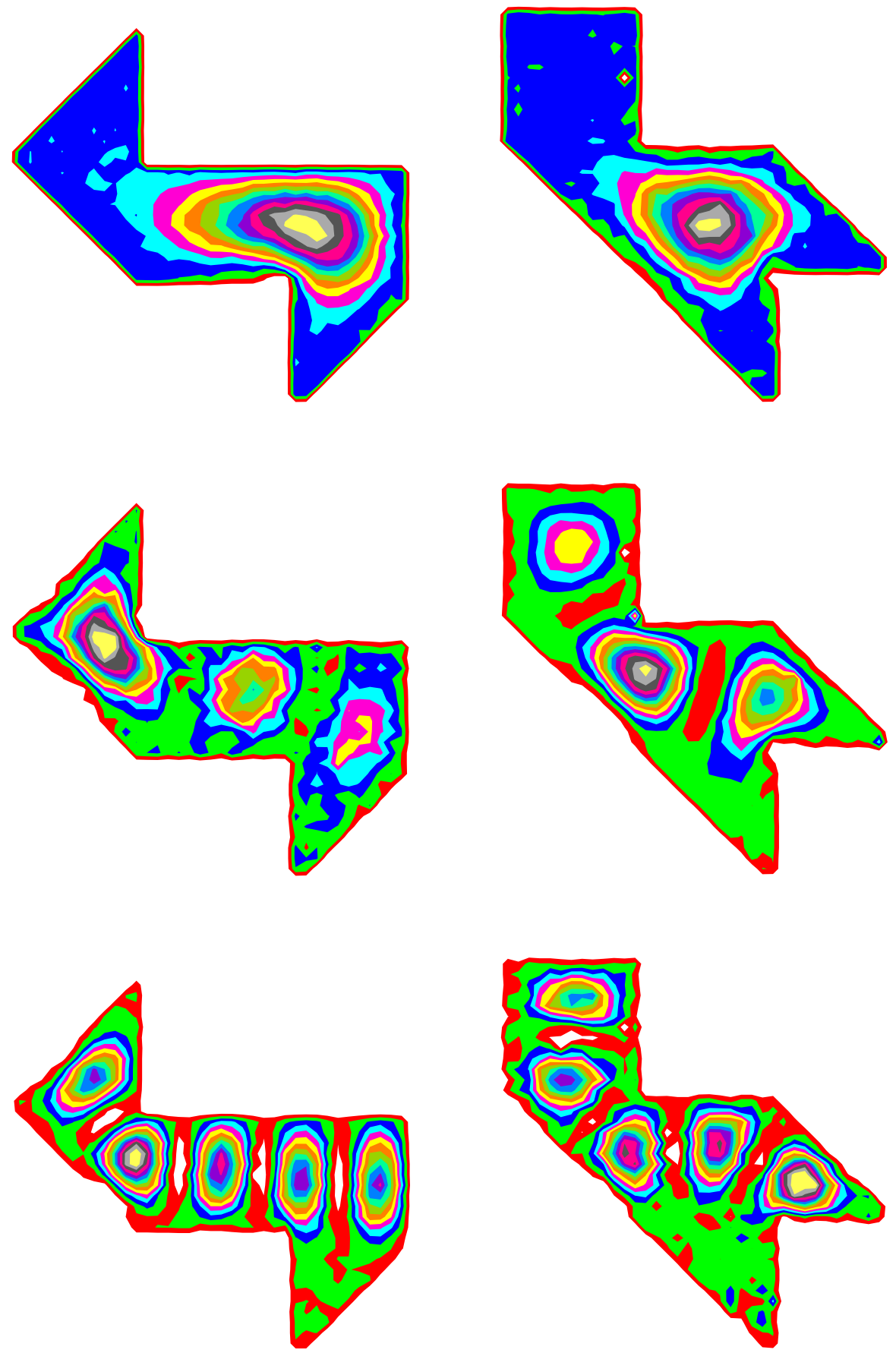

FIG. 9. Eigenfunctions 1,3, and 6 of the $G W W$ isospectral drums, as measured by Sridhar and Kudrolli in their microwave experiments. 

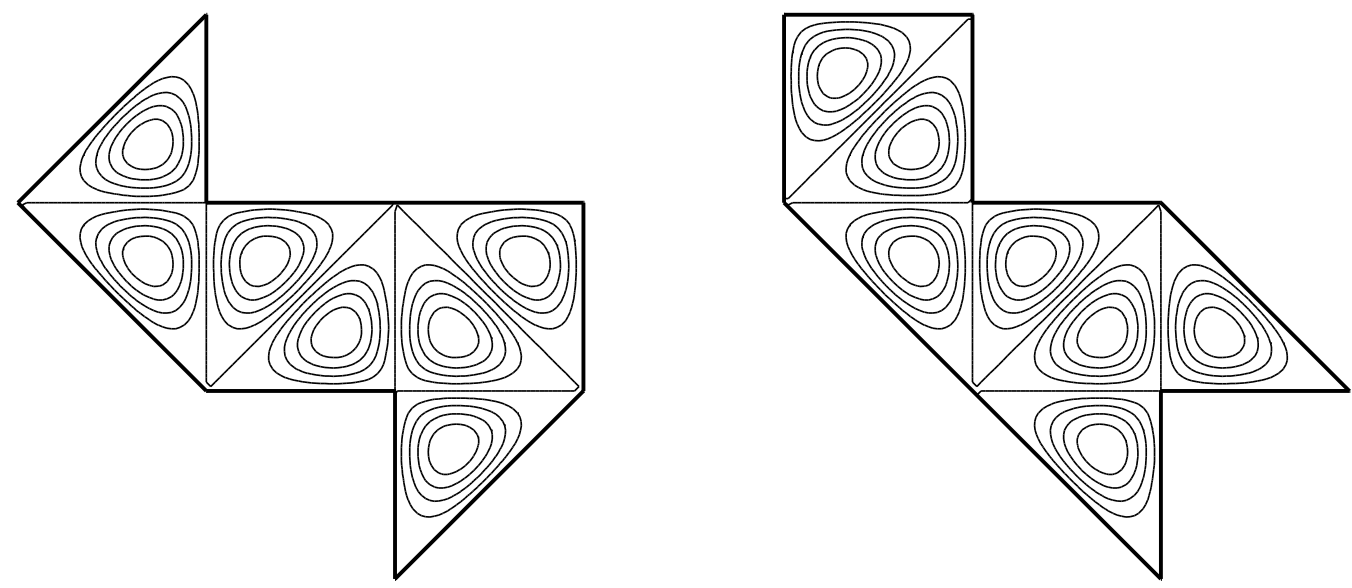

FIG. 10. The ninth mode of the $G W W$ drums. This corresponds to the first mode on a $\left(45^{\circ}, 45^{\circ}, 90^{\circ}\right)$ triangle.

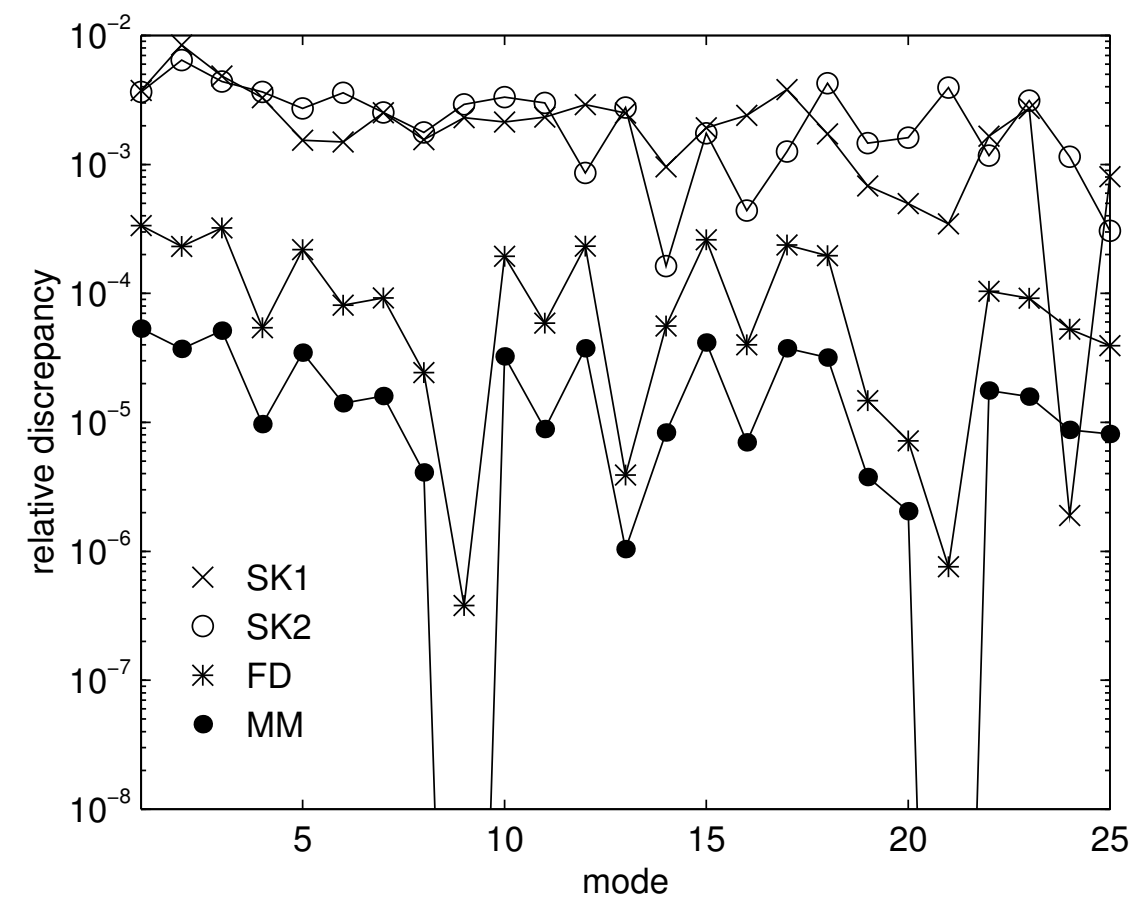

FIG.11. Comparison of our results with other determinations of the spectra. Shown are the two sets obtained by Sridhar and Kudrolli by microwave experiments, and the results of finite differences and mode matching reported by $W u$ et al. 


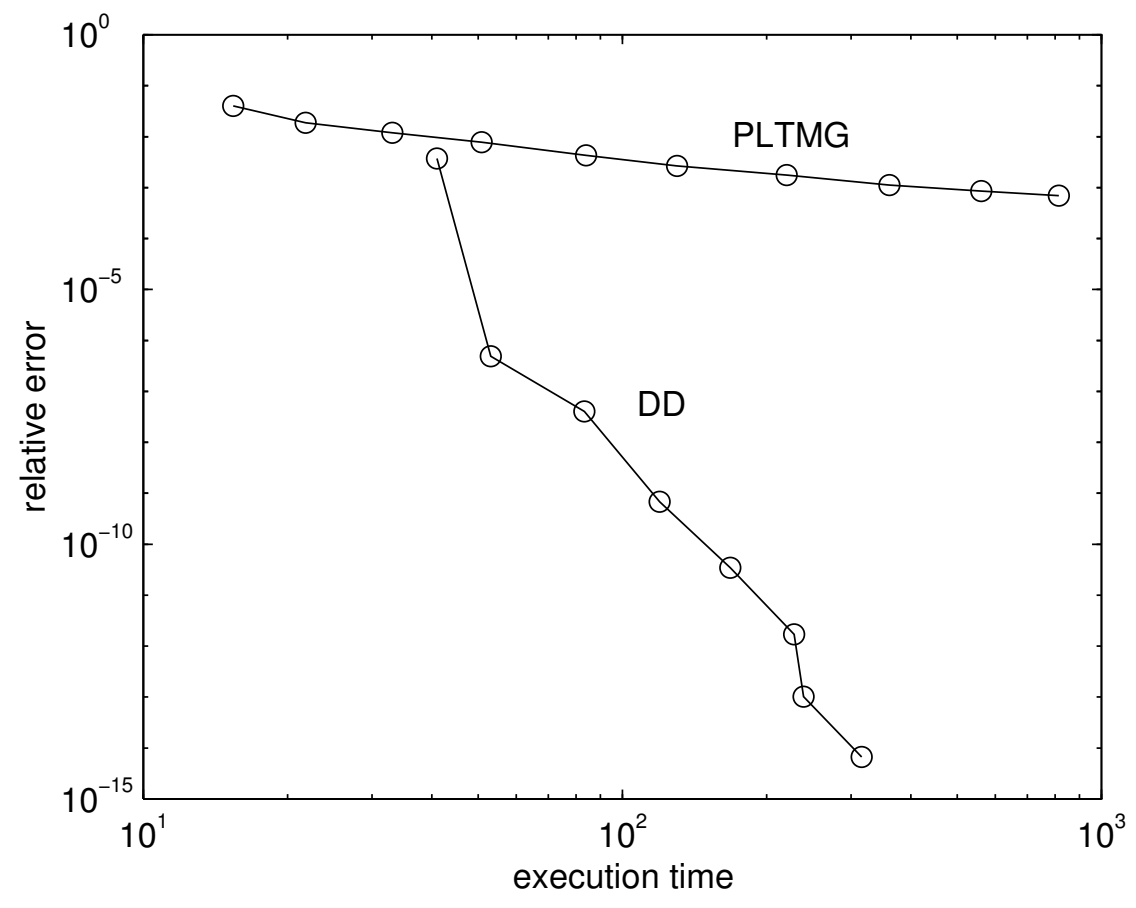

FIG. 12. Comparison of PLTMG with the domain decomposition method. The data are based on the computation of the first eigenvalue of the first $G W W$ drum on a Sun SPARCstation 2.

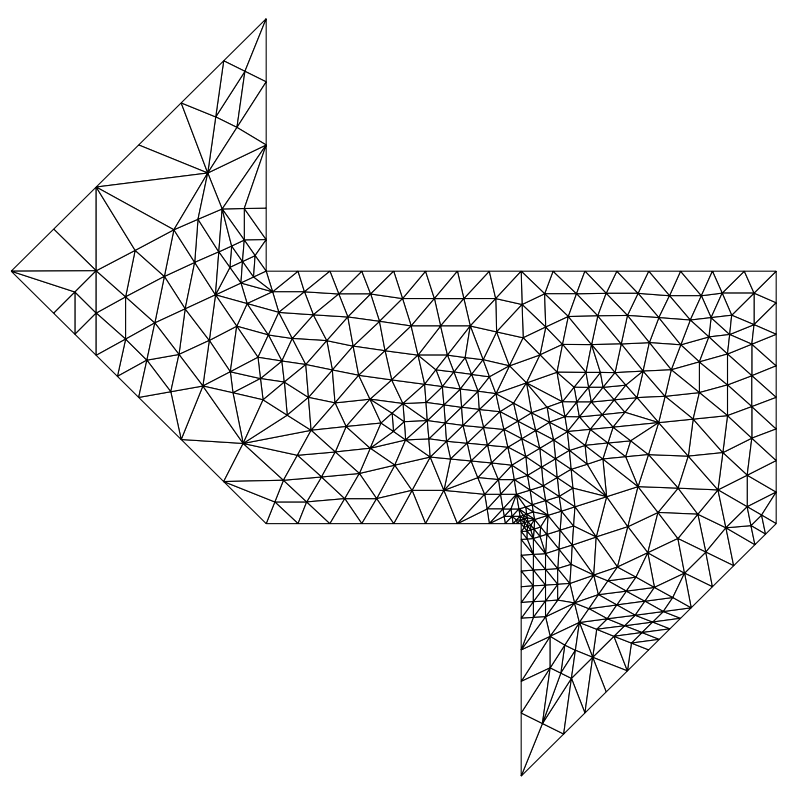

FIG. 13. Adaptive mesh refinement by PLTMG. The mesh is most refined near one of the reentrant corners. 

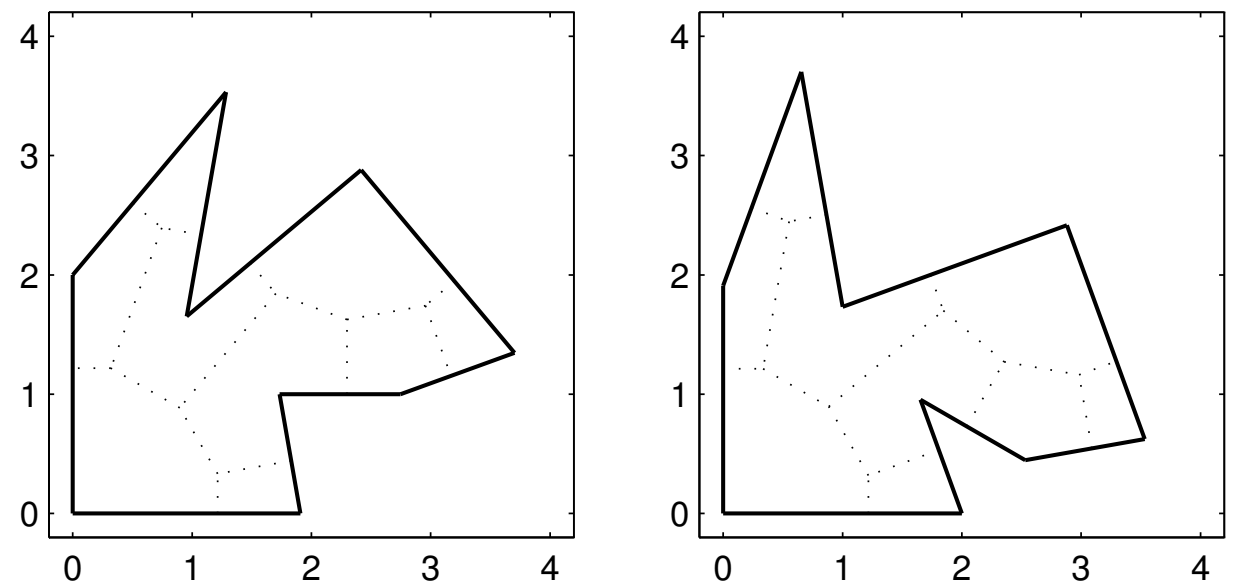

FIG. 14. Two more isospectral regions, and the subdivisions used in the domain decomposition method.

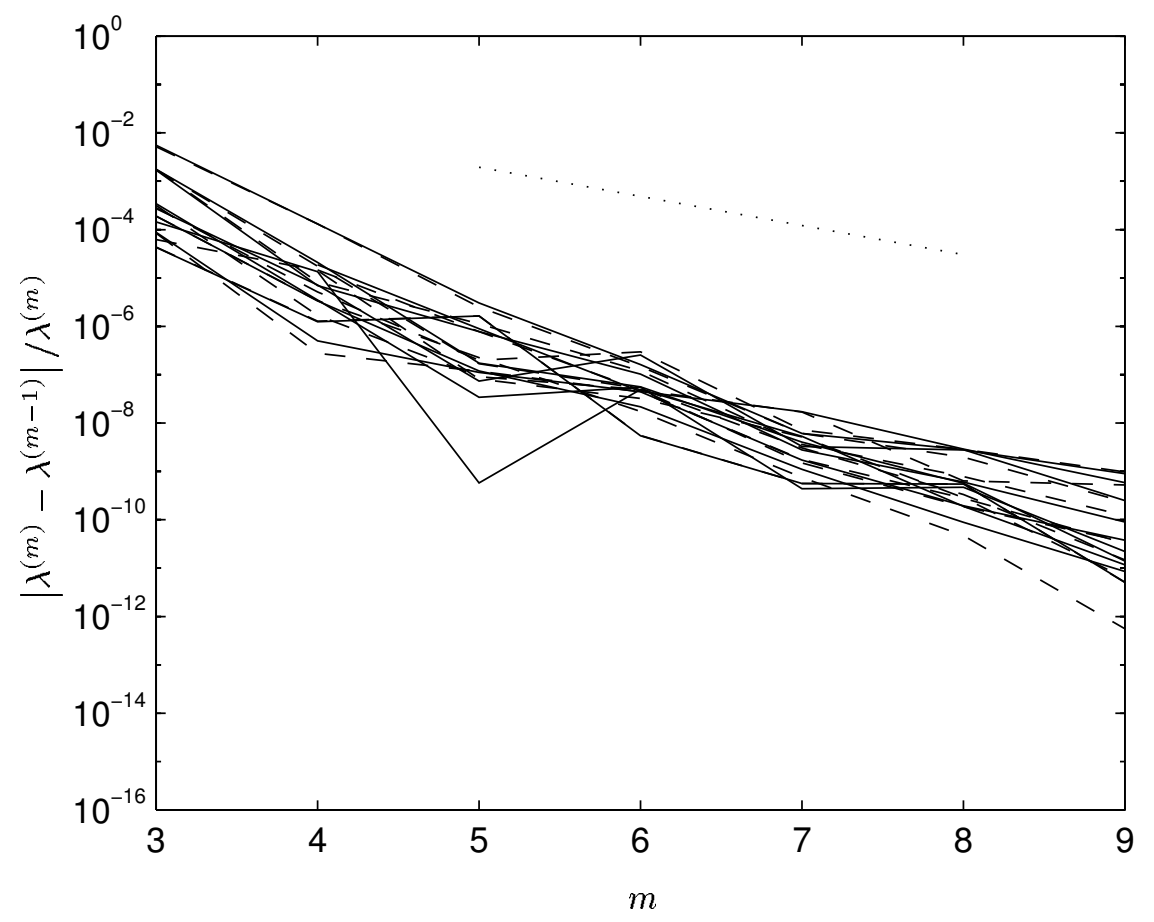

FIG. 15. Convergence of the eigenvalue estimates for the second pair of drums. Solid lines are for the first drum, dashed lines are for the second drum, and the dotted line is a multiple of $\omega^{2 m}$, where $\omega \approx 0.48$. 

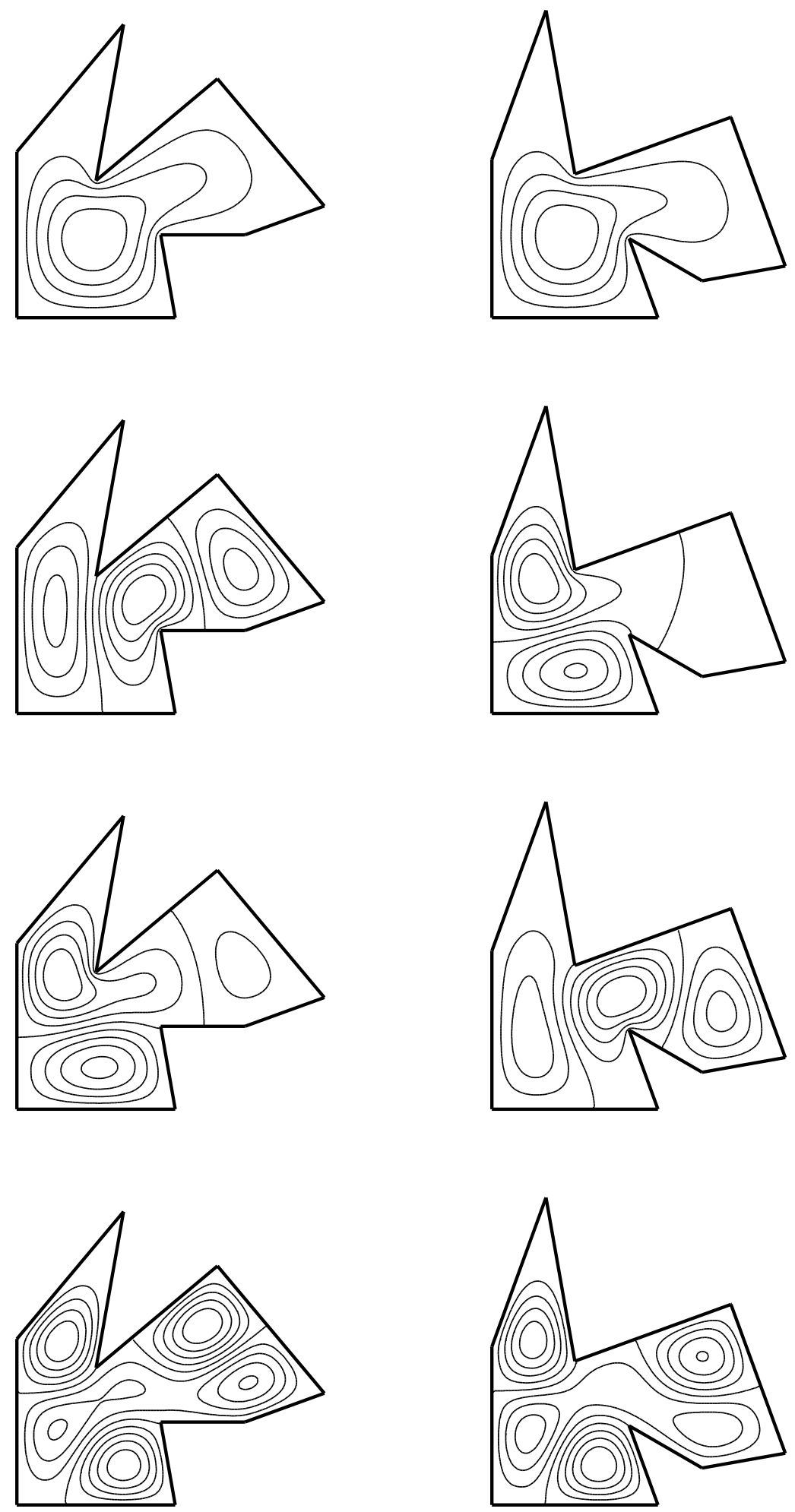

FIG. 16. Eigenmodes 1, 3, 4, and 6 of the second pair of isospectral drums. Modes have unit amplitude, and contours are drawn at $-0.8,-0.6, \ldots, 0.8$. 